\title{
Research of long-term properties of high-strength fiberglasses by means of the built-in FBG sensors
}

\author{
Peter V.Mikheev ${ }^{1}$, Andrey A. Dalinkevich ${ }^{2}$, Sergey A. Gusev ${ }^{1}$, Timofey N. Igonin ${ }^{2}$, Ludmila B. Maksaeva ${ }^{2}$ and Tatyana A. \\ Nenasheva $^{2}$ \\ ${ }^{1}$ Bauman Moscow State Technical University: 2, bd. 1, 2nd Baumanskaya Street, 105005 Moscow, Russia, \\ ${ }^{2}$ Russian academy of sciences A.N. Frumkin Institute of Physical chemistry and Electrochemistry RAS (IPCE RAS) 119991, Leninsky \\ blvd. 31. Moscow, Russia,
}

\begin{abstract}
In this work the possibility of use of the built-in fiber-optical sensors (Bragg's sensors) of deformation for control of a condition of polymeric fiberglasses on epoxy polymer matrix ED-I cured at the increased temperature was investigated. Sensors were inserted in different layers of the layered plastic reinforced by biaxial fiber glass fabric. The package of layers had quasiisotropic structure. The sample made by method of vacuum impregnation was affected by saturated steam $\left(90^{\circ} \mathrm{C}\right)$ within 6 months. Operability of system of measurement has been as a result shown and swelling parameters in different layers of FGRP were recorded. For measurement measuring systems of two types - on a superbright light-emitting diode and on the tunable laser were used.
\end{abstract}

\section{Introduction}

Influence of atmospheric moisture is the most important of climatic factors by which the polymeric composite materials (PCM) at operation of products are affected in nature. As a result of diffusive penetration of water into a polymeric matrix and into the interface between the fiber and matrix in fibrous PCM the part of molecules of water is immobilized on polar groups of polymer, weakening thereby interchain interaction in a matrix and adhesive interaction on limit of the section polymer matrix - fibers. At the same time molecular mobility (effect of plasticization) is released and the relaxation processes leading to restructurings on molecular proceed and supramolecular level.[1,2]

Highly sensitive fiber Bregg grating sensors (FBGsensors) of deformations are successfully used to an assessment of external and internal mechanical and thermal tension in various materials and elements of composite detail [3-7]. Unlike metal FBG strain gages sensors do not rust and therefore they can be applied in the damp hot environment. Besides their accuracy is higher than at wire strain gages.

The purpose of work consisted in clarification of opportunities to use fiber-optical sensors of Bragg (FBGsensors) for studying micromechanical moist arising in PCM at the accelerated heat-moist influence which is widely applied to modeling of climatic aging of PCM.

The problem of influence of moisture on fibreglass is important, so this material is perspective for application in networks of water supply and oil pipelines. Fiberglass pipes of less steel are subject to corrosion.

\section{Experimental}

For production of a sample biaxial fiber glass fabric is used (specification TU 1916-033-61664530-2013, produced by JSC Prepreg-SKM with area density of 860 $\mathrm{g} / \mathrm{m} 2$ and the direction of fibers $0^{\circ} / 90^{\circ}$.

Multiaxial fabric has been chosen with the purpose to imitate the pipes made by winding. The quasiisotropic structure is chosen as the most applicable.

Matrix epoxy - ED-I (specification TU B3-774-2010) consist of composition from ED-20 epoxy (100 $\mathrm{m}$ of $\mathrm{h}$ ) of IMTGFA hardener (isomethyltetrahydrophthalic anhydride, $20 \mathrm{~m}$ of $\mathrm{h}$ ) and the accelerator unitary enterprise 606/2 (2.4,6, Tris (dimethylaminomethyl) of phenol. This type of a matrix is used in the industry and differs in high resistance to moisture. Samples of binding have been made by method of filling of images in a form. And then their mechanical properties have been defined. Before filling binding was maintained under vacuum not to allow hit of vials of air in a sample.

FGRP samples on the basis of biaxial fabric and epoxy resin ED-I produced by an infusion method. Specimen was cured at $120^{\circ} \mathrm{C}$.

Completeness of hardening polymer matrix was controlled by method of the differential scanning calorimetry, glass temperature of the sample cured binding (without reinforcing fibres ) has made $132{ }^{\circ} \mathrm{C}$. (Figure 1).

FBG sensors have been located between layers at a different depth of a sample. When laying layers, the 
second layer has been turned by 45 degrees to create structure close to isotropic structure.

Previously the method for output of optical sensors out of plastic has been worked out. For this purpose samples of fiberglass plastic with optical fibre but without sensors were prepared. So we picked up a way to protect the optical fiber from being damaged at the edges of the plastic and at the junction of the measuring fiber with patch cords. Initially the stresses arising during cooling of the finished fiberglass plate either destroyed the sensor or bent it. This distortion leads to the loss of the optical signal below the level of sensitivity threshold of the system based on high-brightness LEDs (the method of Bauman Moscow State Technical University, BMSTU).

After picking up a set of heat shrinking tubing the problem was solved. At the same time on the plates with an optical fiber without sensors was selected the temperature for preserving patch cords connectors from damage or from resin casting.

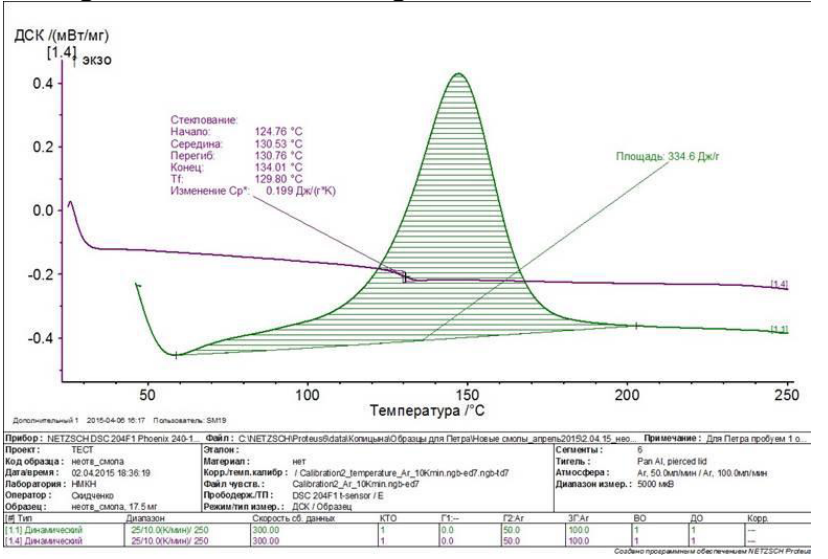

Figure 1. Chart of hardening of epoxy matrix

Sensors have been integrated into a sample only when was obtained the attenuation of a light signal accepted for measurements.

Mechanical properties of epoxy binding have been checked on standard samples "a dog bone". Results are given in Tab. 1. Binding has shown fragile linear behavior up to destruction.

Table 1 Properties of polymeric binding

\begin{tabular}{|c|l|c|c|}
\hline № & \multicolumn{1}{|c|}{ Property } & Value & $\begin{array}{c}\text { Variation } \\
\text { factor, \% }\end{array}$ \\
\hline 1 & Elasticity module, GPA & 2.84 & 1.75 \\
\hline 2 & Strength, MPa & 38.3 & 14.5 \\
\hline 3 & ultimate strain,\% & 1.4 & 15.7 \\
\hline 4 & Dencity, gm/cm 3 & 1.22 & 5.0 \\
\hline
\end{tabular}

Parameters of FBG - sensors optical (produced by "Inversion Sensor") were the following:

top layer (marking No. 1341) Wavelength second layer (marking No. 13801542,28

second layer (marking No. 1380) Wavelength 1550,23 center (marking No. 1340) Wavelength 1554,06

The arrangement of sensors is given in layers on rice.2. On Fig. 3 process of laying of optical fibers with sensors between layers of industrial fiber-glass fabric before impregnation of a sample binding is shown.

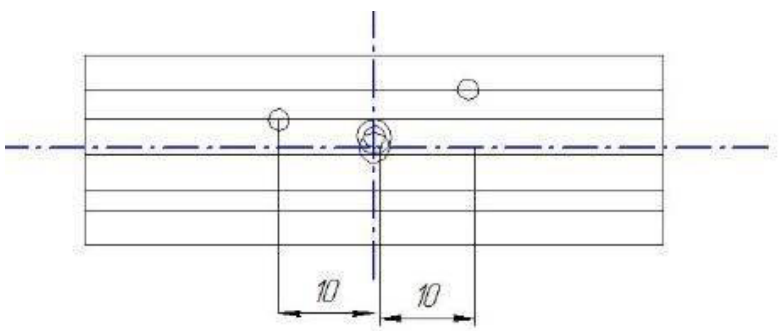

Figure 2. The scheme of an arrangement of sensors in sample layers

The sensors located in different layers have been carried on width not to create distortion of structure. For production of samples the following devices were used:

- Installation of vacuum impregnation Vacmobile INFUSION 28/2 (New Zealand);

- France Etuves XXL04.5 furnace (France).

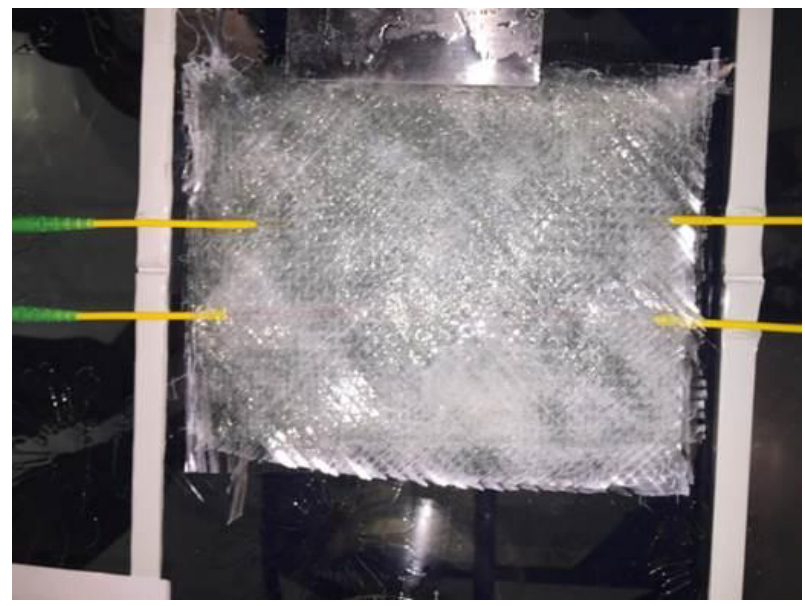

Figure 3. Process of laying of sensors between fiber glass fabric layers

For determination of temperature of vitrification binding the differential scanning calorimeter Netzch DSC 204 F1 Phoenix (Germany) was used.

For determination of mechanical properties of a sample of the witness the universal test equipment ZwickRoel Z-100 (Germany) was used.

The endurance of samples was carried out in the certified thermo-moist camera Quantel the MHK $408 \mathrm{CL}$ model (Singapore) Figure 4.

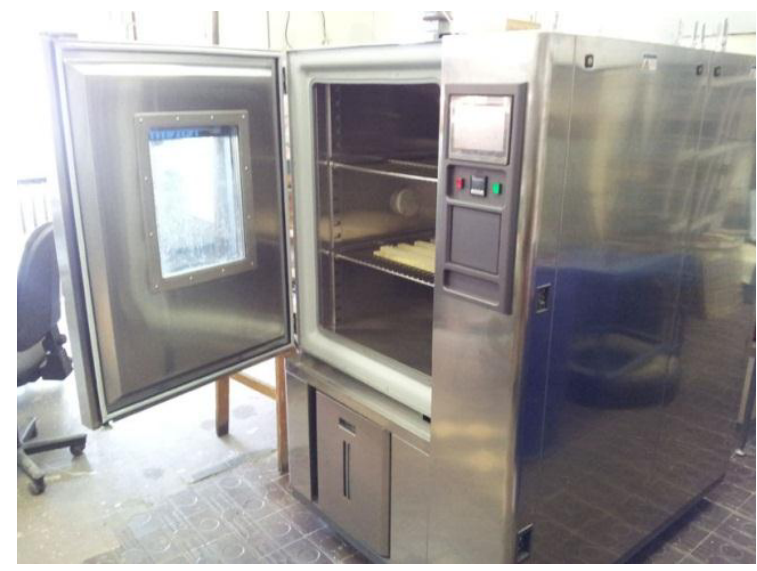

Figure 4. Thermo-moist camera

Experimental measurements were made of the measuring system developed in NOC $\mathrm{s}$ "Photonics and 
IK-equipment" of BMSTU and the Astro A312 device of production "Inversion Sensor". These measurements received by means of both systems corrections for their use in one measuring system are compared among themselves.

The measurement system constructed in BMSTU was based on source of the white light, technically more simply, but the Astro system based on the tunable laser has big sensitivity and bigger speed. 5 .

One of optical spectra of the sensor is given in Figure

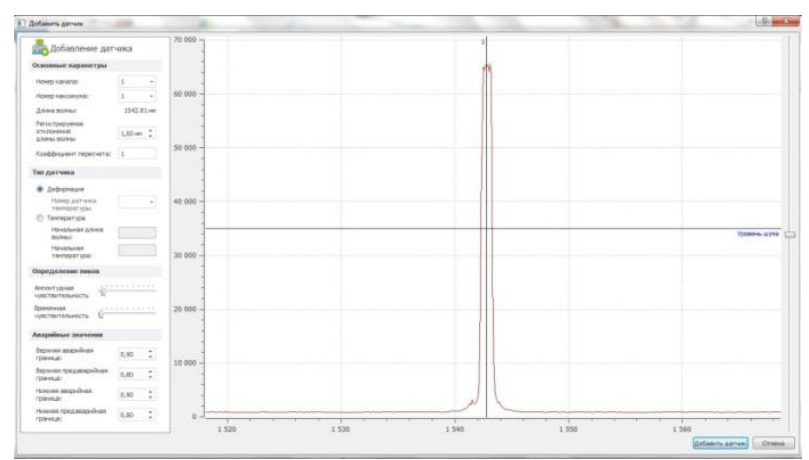

Figure 5. The example of an optical signal from the FBGsensor received on the equipment Astro.

The scheme of sensors arrangement is provided in on Fig 2 and in Table 1. To reveal spatial unevenness of penetration of water into a sample, sensors were put on different depth (layers 1, 2 and 3). The increased temperature of hardening of polymeric binding has demanded special protection of optical fibers in places of an exit from FGRP.

Table 1. Arrangement of sensors in layers of FGRP

\begin{tabular}{|c|c|}
\hline Material & SM-42019 $\left(+0 / 90^{\circ}\right)$ \\
\hline Layer № & laying \\
\hline 1 & SM-42019 $\left(+0 / 90^{\circ}\right)$ \\
\hline FBG-sensors 1 & Measuring layer \\
\hline 2 & SM-42019 $\left(+45 /-45^{\circ}\right)$ \\
\hline FBG-sensors 2 & Measuring layer \\
\hline 3 & SM-42019 $\left(+0 / 90^{\circ}\right)$ \\
\hline FBG-sensors 3 & Measuring layer \\
\hline 4 & SM-42019 $\left(+0 / 90^{\circ}\right)$ \\
\hline 5 & SM-42019 $\left(+45 /-45^{\circ}\right)$ \\
\hline 6 & SM-42019 $\left(+0 / 90^{\circ}\right)$ \\
\hline
\end{tabular}

Dynamics of change of weight of a sample due to absorption of moisture is shown in Figure 7. In the same Figure the schedule of measurement of deformations is shown.

Special attention has been paid to protection of connectors against the increased temperature and humidity. Influence of hot water was so strong that all factory marking of sensors has been washed away.

The doubling sample on the same epoxy matrix ED-I and fabrics, but without sensors has been in parallel made. Samples for determination of density (hydrostatic weighing) and some physicomechanical characteristics (Tab. 2) have been cut out from FGRP plates.

Measurements of mechanical properties of a doubling sample have shown that it possesses good mechanical properties.

The doubling sample has been cutted near the sensor location. The photo on Fig. 6 shows the end face of sensor. It is visible that there are no intervals between the sensor and matrix, and the structure of a composite is not broken.

Table 2. Properties of the made fibreglass

\begin{tabular}{|c|l|c|}
\hline$№$ & \multicolumn{1}{|c|}{ Property } & Value \\
\hline 1 & Elasticity module at a bend, GPA & 21,7 \\
\hline 2 & Elasticity module at stretching, GPA & 20,6 \\
\hline 3 & Strength at bending, MPa & 397 \\
\hline 4 & Strength at shift, MPa & 38,3 \\
\hline 5 & Density of plastic, g/cm3 & 1,9 \\
\hline 6 & $\begin{array}{l}\text { Volume fraction of the reinforcing } \\
\text { fibers, \% }\end{array}$ & 55 \\
\hline 7 & $\begin{array}{l}\text { Density of of the used polymer matrix, } \\
\mathrm{g} / \mathrm{cm} 3\end{array}$ & 1,22 \\
\hline
\end{tabular}

The FGRP sample containing sensors has been subjected to heat-moist aging in a test climatic chamber at the temperature of $80{ }^{\circ} \mathrm{C}$ and relative humidity of $95 \%$. Accuracy of maintenance of parameters of tests made $\pm 1^{\circ} \mathrm{C}$ for temperature and $\pm 1,5 \%$ for humidity.

Moisture content in the studied sample of FGRP was controlled by method of periodic weighing with an accuracy of 10-3 g.

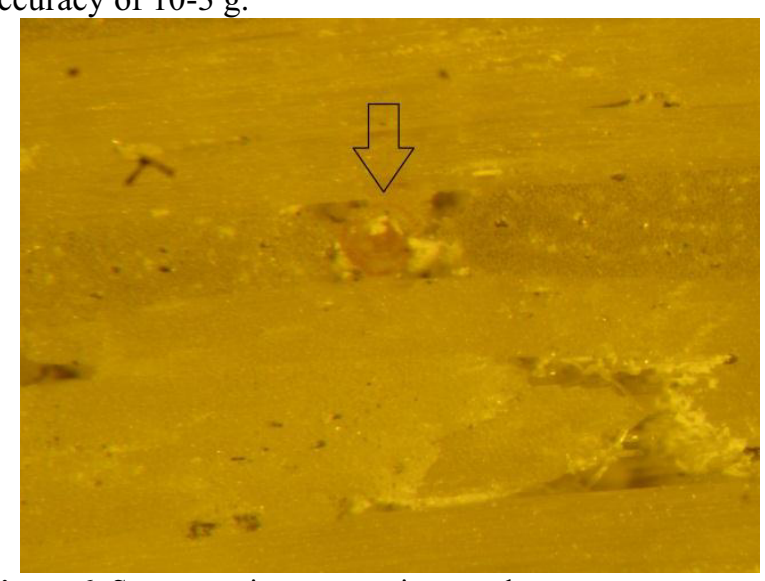

Figure 6. Sensor cut in a composite sample

\section{Results and discussion}

From Fig. 7 it is visible that sorption of vapors of water FGRP has abnormal character and does not submit to Fick's law. Existence of a maximum and step on kinetic curve sorption demonstrates course in a model of relaxation processes, i.e. change of internal microstructural tension and according to deformations which, in turn, are caused by diffusive and sorption penetration and interaction of water with polymer [8-11].

High sensitivity to temperature and deformation of a FBG-sensors also allow to use it as a sensitive element of the measuring system. Measuring properties of a FBGsensors depend on effective index of refraction and on the 
lattice period on a formula (1). And the period of a lattice is directly connected with its deformation:

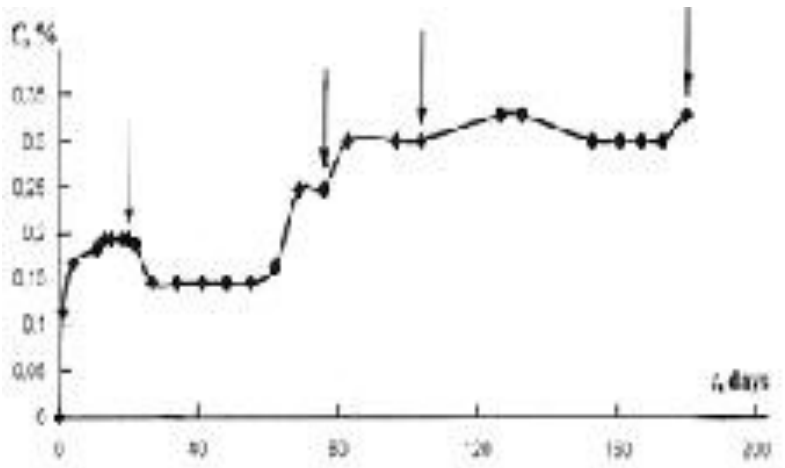

Figure 7. Water sorption by fiberglass. Arrows showed the moments of measurement of deformations

$$
\Delta \lambda_{B}=2 \cdot\left(\lambda \frac{\partial n_{e f}}{\partial l}+n_{e f} \frac{\partial \lambda}{\partial l}\right) \cdot \Delta l+2 \cdot\left(\lambda \frac{\partial n_{e f}}{\partial T}+n_{e f} \frac{\partial \lambda}{\partial T}\right) \cdot
$$$$
\Delta T
$$

The first part of equation gives value of shift of wavelength depending on deformation (lengthening), the second depending on temperature. Dependence of the central reflected wavelength on deformation can be also presented in the following form [5]:

$$
\Delta \lambda_{B}=\lambda_{B} \cdot\left(1-p_{e}\right) \cdot \varepsilon_{z}
$$

where $p_{e}$ - the constant of deformation of optical fiber defined from the following expression

$$
p_{e}=\frac{n_{e f}^{2}}{2} \cdot\left[p_{12}-v \cdot\left(p_{11}-p_{22}\right)\right]
$$

where $p_{11}$ и $p_{12}$ - Pokkels's coefficients in a tensor of optical tension, - Poisson's coefficient. For standard fiber $p_{11}=0,113, p_{12}=0,252, \quad=0,16$ and $n_{e f}$ $=1,4682$ [6]

Proceeding from these values sensitivity for wavelength $B_{B}=1550$ nanometers make 12 nanometers $/ \%$.

Deformation of sensors after endurance in water calculated on the basis of (3) is given in Table 3 .

Measurements were taken once a month and until optical measurement the sample was stored in the damp atmosphere. Thus we interfered with probable deformations when drying.

Table 3. Deformation increment from the moment of GFRP producing

\begin{tabular}{|l|c|c|c|c|}
\hline $\begin{array}{l}\text { Deformation } \\
\text { in time, } \\
\%, \%\end{array}$ & 15.07 .15 & 10.09 .15 & 09.10 .15 & 23.12 .15 \\
\hline Upper layer & 0,007 & $-0,017$ & $-0,014$ & $-0,074$ \\
\hline Second layer & 0,013 & 0,003 & 0,007 & $-0,001$ \\
\hline Middle layer & 0,015 & 0,003 & 0,009 & $-0,006$ \\
\hline
\end{tabular}

In Figure 8 changes of deformation of layers in the form of the histogram are presented.

In drawing it is well visible that external layers absorb water stronger than internal. But during such a long period (6 months), process becomes stationary and does not lead to destruction of a composite.

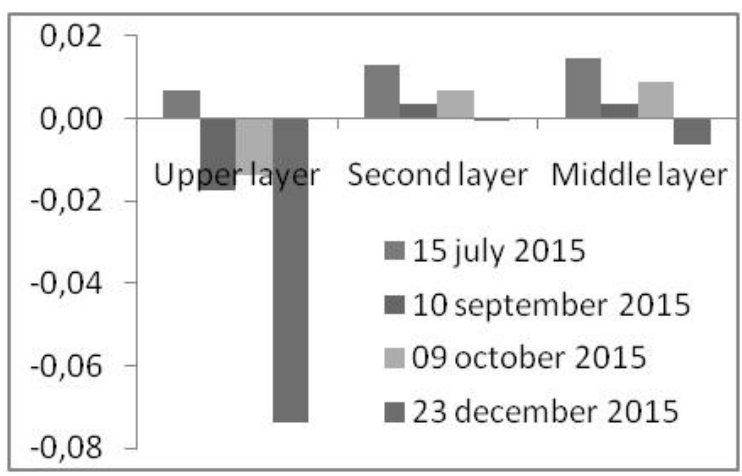

Figure 8. Deformations of layers of fibreglass at endurance in the damp atmosphere.

\section{Conclusions}

The possibility of fixing of thermomoist deformations in fiberglass of quasiisotropic structure is shown for a long time. Two types of measuring equipment are used: the BMSTU experimental system on the basis superbright light-emitting diode and the Astro A312 system on the basis of the tunable laser.

Deformations of swelling in different layers of fiberglass are recorded. In general epoxy resin ED-I has shown high resistance at influence of temperature and moisture.

The equipment of the built-in sensors can be used for a prediction of resource of the polymeric detail working in the conditions of long influence of water. Bragg's sensors are not subject to corrosion and therefore are suitable for such task. In our research ED-I epoxy has shown high resistance to hot water

Certain results of this work were obtained as a part of research works upon Agreement with the Ministry of Science and Education of the Russian Federation concerning the provision of grant № 14.577.21.0103 dated 16.09. 2014. The research project ID RFMEFI57714X0103

\section{References}

1. A. Cusano, A. Cutolo, J. Albert. "Fiber Bragg Grating Sensors: Recent Advancements, Industrial Applications and Market Exploitation", Editors, Bentham Science Publishers, 2010, PP. 35-52.

2. A.A. Dalinkevich, K.Z. Gumargalieva, S.S. Marakhovsky, A.V. Soukhanov, Modern Basalt Fibrous Materials and Basalt Fiber-Based Polymeric// JOURNAL OF NATURAL FIBERS, 2009,VL 6, № 3, P 248-271,

3. Yu.N Kulchin. The distributed fiber-optical sensors and measuring networks Vladivostok// Dalnauka, 1999, PP. 115-118.(In Russian)

4. M. Ramakrishnan, G. Rajan, Y. Semenovaand, G. Farrell, Overview of Fiber Optic Sensor Technologies forStrain/Temperature Sensing Applications in 
Composite Materials, Sensors// 2016, № 16, V. 99; doi:10.3390/s16010099

5. S. Hannuscha, M. Stockmanna, J. Ihlemanna, Experimental method for residual stress analysis with fibre Bragg grating sensors, Materials Today: Proceedings 3 ( 2016 ), PP. 979 - 982

6. K.Yükseln, D. Pala, Analytical investigation of an ovelinterrogation approach of fiber Bragg grating sensors using Optical Frequency Domain Reflectometry// Optics and Lasers in Engineering 2016, № 81, PP.119-124

7. D. Abbaneo and other, Fiber Bragg Grating(FBG) sensors as flatness and mechanical stretching sensors//, Nuclear Instrumentsand Method sin Physics Research, 2016, V.824, PP. 493-495

8. P.V. Mikheev, A.V. Artemyev, V.A. Lazarev, A.B. Pnev, V.A. Nelyub, "Experience of creation of system of the built-in control of a condition of a design on the basis of fiber-optical the breggovskikh of sensors", the Collection of reports of the All-Russian conference on tests and researches of properties of the materials TEST MAT - 2013. Publishing house: All-Russian Research Institute of Aviation Materials (Moscow.) 2013, PP. 28-29. ( In Russian)

9. V.V. Shishkin, A.E. Churin, D.S. Kharenko, I.S. Shelemba, "System of monitoring of the bearing designs of a football arena on the basis of fiber-optical sensors"// Foton-Express, 2013, № 6, V.110, PP. 22-23 (2013) (In Russian)

10. V.V. Shishkin, A.E. Churin, D.S. Kharenko, M.A. Zheleznova, I.S.Shelemba, "Structural health monitoring system of soccer arena based on optical sensors", Proc. SPIE 9157, 23rd International Conference on Optical Fibre Sensors, 9157D3 (2 June 2014); doi: 10.1117/12.2071269, (2014)

11. B.S. Sarbayev, A.A. Smerdov, L.P. Tairova, V.A. Seleznyov, S.V. Sokolov, G.Ya. Buymistryuk, V.I. Izotov, A.M. Rogov, "Research of the deformed condition of designs from composite materials by means of fiber optical sensors"// Bulletin of Bauman Moscow State Technical University. 2011. N.SP, PP. 39-51. (In Russian)

12. E. Bar, Constructional properties of plastic (physical and chemical bases of application). The lane with English M., "Chemistry", 1967. PP. 231-235. (In Russian

13. Yu.A. Mikhaylin Fibrous polymeric composite materials in equipment. - SPb.: "Profession", 2013. 752 pages. (In Russian)Modern physical methods of research of polymers. Under the editorship of Slonimsky G. L. M, "Chemistry", 1982. PP. 198-208. (In Russian) 